\title{
$Z^{\prime}$ signals in polarised top-antitop final states at the LHC
}

\section{Lorenzo Basso}

Physikalisches Institut, Albert-Ludwigs-Universität Freiburg

E-mail: lorenzo.basso@physik.uni-freiburg.de

\section{Ken Mimasu*}

School of Physics \& Astronomy, University of Southampton

E-mail: k.mimasulsoton.ac.uk

\section{Stefano Moretti}

School of Physics \& Astronomy, University of Southampton

Particle Physics Department, Rutherford Appleton Laboratory

E-mail: s.moretti@soton.ac.uk

\begin{abstract}
We study the sensitivity of top pair production at the Large Hadron Collider (LHC) to the nature of an underlying $Z^{\prime}$ boson, including full tree level standard model background effects and interferences while assuming realistic final state reconstruction efficiencies. We demonstrate that exploiting asymmetry observables represents a promising way to distinguish between a selection of benchmark $Z^{\prime}$ models due to their unique dependences on the chiral couplings of the new gauge boson.
\end{abstract}

36th International Conference on High Energy Physics

4-11 July 2012

Melbourne, Australia

${ }^{*}$ Speaker. 


\section{Introduction}

$Z^{\prime}$ bosons are a ubiquitous feature of theories beyond the Standard Model (SM) arising from various BSM scenarios such as $U(1)$ gauge extensions of the SM motivated by supersymmetry or grand unified theories, Kaluza-Klein excitations of SM gauge fields or excitations of composite exotic vector mesons in technicolor theories to name a few.

Typically, such resonances are searched for at hadron colliders via the Drell-Yan (DY) production of a lepton pair, i.e., $p p(\bar{p}) \rightarrow\left(\gamma, Z, Z^{\prime}\right) \rightarrow \ell^{+} \ell^{-}$, where $\ell=e, \mu$. The Tevatron places limits on the $Z^{\prime}$ mass, $M_{Z^{\prime}}$, at around $1 \mathrm{TeV}$ [1] (for a sequential $Z^{\prime}$ ) while the latest LHC limits lie around 2.3 $\mathrm{TeV}$ [2] from this channel. Several phenomenological studies on how to measure the $Z^{\prime}$ properties and couplings to SM particles in this clean DY channel have been performed.

These proceedings summarise a recently published paper [3] addressing the use of the topantitop final state, i.e., $p p(\bar{p}) \rightarrow\left(\gamma, Z, Z^{\prime}\right) \rightarrow t \bar{t}$, to probe these $Z^{\prime}$ properties. While it may not have as much 'discovery' scope as the DY channel, owing to the large QCD background combined with the complex six-body final state and the associated reconstruction efficiency, it remains important to extract the couplings of new physics to the top quark. Furthermore, the fact that the top decays before hadronising, transmitting spin information to its decay products, allows for the definition of spin asymmetry observables which provide an extra handle on $Z^{\prime}$ couplings not present in nondecaying final states.

We study the scope of the LHC to profile a $Z^{\prime}$ boson mediating $t \bar{t}$ production, in both standard kinematic variables as well as spatial/spin asymmetries, by adopting some benchmark scenarios for several realisations of the sequential, Left-Right symmetric and $E_{6}$ based $Z^{\prime}$ models (specifically, the same as those in [4]). Specifically, the issue of distinguishability of various models using these observables is addressed.

\section{Asymmetries and $Z^{\prime}$ couplings}

We define the asymmetry observables considered with the aim of determining their power to discriminate between $Z^{\prime}$ s. We refer the reader to our paper for a more detailed discussion on these as well as the selection of benchmark models, statistical uncertainties and definitions of significance. This study investigated charge (spatial) and spin asymmetries and their dependence on top couplings to profile and distinguish the models considered.

A selection of charge asymmetry variables were investigated with the most sensitive found to be $A_{R F B}$, defined by the rapidity difference of the top and antitop, $\Delta y=\left|y_{t}\right|-\left|y_{\bar{t}}\right|$, while also cutting on the boost of the $t \bar{t}$ system. This increases the contribution from the $q \bar{q}$ initial state by probing regions of higher partonic momentum fraction, $x$, where its parton luminosity is more important:

$$
A_{R F B}=\left.\frac{N(\Delta y>0)-N(\Delta y<0)}{N(\Delta y>0)+N(\Delta y<0)}\right|_{\left|y_{t i t}\right|>\mid y_{t i}^{c u t}} .
$$

The two spin asymmetries considered, termed double $(L L)$ and single $(L)$, are defined as follows:

$$
A_{L L}=\frac{N(+,+)+N(-,-)-N(+,-)-N(-,+)}{N_{\text {Total }}} \quad ; \quad A_{L}=\frac{N(-,-)+N(-,+)-N(+,+)-N(+,-)}{N_{\text {Total }}}
$$


where $N$ denotes the number of observed events and its first(second) argument corresponds to the helicity of the top (anti)quark. These observables are alternatively known as the spin correlation and spin polarisation asymmetries respectively and can be extracted as coefficients in differential angular distributions of the top decay products.

Defining a generic neutral current interaction with the Feynman rule

$$
i \gamma^{\mu}\left(g_{V}-g_{A} \gamma^{5}\right) \equiv i \gamma^{\mu}\left(P_{L} g_{L}+P_{L} g_{R}\right)
$$

we can calculate the dependence of the asymmetries in terms of its vector and axial couplings $g_{V}$ and $g_{A}$ or alternatively, the left and right handed couplings $g_{L}$ and $g_{R}$. These were obtained using the helicity formulae from [6] in the case of the spin asymmetries (also derived independently with the guidance of [7]), where $i$ and $t$ denote the initial state and the top quark respectively:

$$
\begin{aligned}
A_{F B}^{i} & \propto g_{V}^{i} g_{A}^{i} g_{V}^{t} g_{A}^{t} \\
& \equiv\left(\left(g_{R}^{i}\right)^{2}-\left(g_{L}^{i}\right)^{2}\right)\left(\left(g_{R}^{t}\right)^{2}-\left(g_{L}^{t}\right)^{2}\right), \\
A_{L L}^{i} & \propto\left(\left(g_{V}^{i}\right)^{2}+\left(g_{A}^{i}\right)^{2}\right)\left(3\left(g_{A}^{t}\right)^{2} \beta^{2}+\left(g_{V}^{t}\right)^{2}\left(2+\beta^{2}\right)\right) \\
& \equiv\left(\left(g_{L}^{i}\right)^{2}+\left(g_{R}^{i}\right)^{2}\right)\left(\left(g_{L}^{t}+g_{R}^{t}\right)^{2}+2\left(\left(g_{L}^{t}\right)^{2}-g_{L}^{t} g_{R}^{t}+\left(g_{R}^{t}\right)^{2}\right) \beta^{2}\right), \\
A_{L}^{i} & \propto\left(\left(g_{V}^{i}\right)^{2}+\left(g_{A}^{i}\right)^{2}\right) g_{A}^{t} g_{V}^{t} \beta \\
& \equiv\left(\left(g_{R}^{i}\right)^{2}+\left(g_{L}^{i}\right)^{2}\right)\left(\left(g_{R}^{t}\right)^{2}-\left(g_{L}^{t}\right)^{2}\right),
\end{aligned}
$$

for a neutral gauge boson exchanged in the $s$-channel, with $\beta=\sqrt{1-4 m_{t}^{2} / \hat{s}}$. The charge asymmetry depends on the product of the vector and axial couplings of both the initial and final state particle and can only be generated when all of these are non-vanishing. Alternatively it is a function of the relative magnitudes of their right and left handed couplings. For the spin asymmetries, $A_{L L}$ depends on the couplings in a similar way to the total cross section becoming maximal in the limit $\beta \rightarrow 1$, while $A_{L}$ is only non-vanishing for non-zero vector and axial couplings of the final state tops and is additionally sensitive to their relative sign. This is equivalent to measuring their relative handedness but only for the final state. Note that $A_{L}$ allows for the direct measurement of this feature of the top couplings while this information is lost in the charge asymmetry due to the identical dependence on the initial state couplings.

With these unique coupling dependences, it is our aim to show that asymmetries can provide extra information to distinguish $Z^{\prime}$ models and ultimately contribute to extracting the couplings of an observed neutral resonance.

\section{Results}

We present a selection of results profiling the spatial and spin asymmetry distributions of the benchmark $Z^{\prime}$ models compared to the SM including interference effects. The set of benchmarks are split into two categories: those with a vanishing vector or axial coupling (the $E_{6}$ models with the 'B-L' generalised left-right symmetric model) are classed as the ' $E_{6}$ ' type while the rest, with both couplings non-zero, are referred to as the 'generalised' models. The variables described in 
section 2 were computed as a function of the $t \bar{t}$ invariant mass within $\Delta M_{t \bar{t}}=\left|M_{Z^{\prime}}-M_{t \bar{t}}\right|<500$ $\mathrm{GeV}$ and compared to the tree-level SM predictions. The code exploited for our study is based on helicity amplitudes, defined through the HELAS subroutines [8], and built up by means of MadGraph [9]. CTEQ6L1 [10] Parton Distribution Functions (PDFs) were used, with the factorisation/renormalisation scale at $Q=\mu=2 m_{t}$. VEGAS [11] was used for numerical integration.
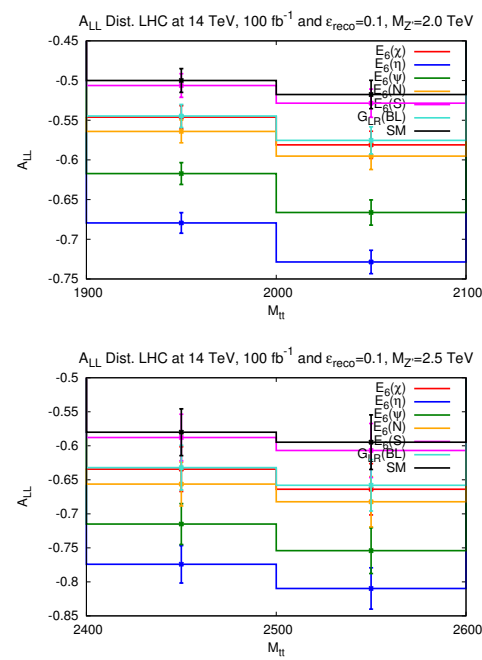
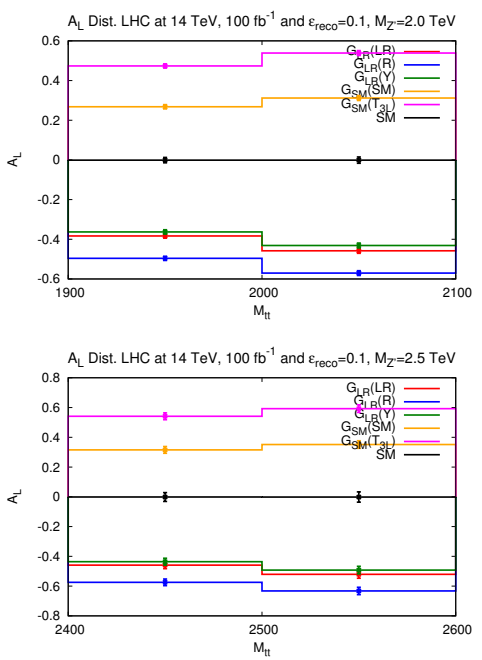
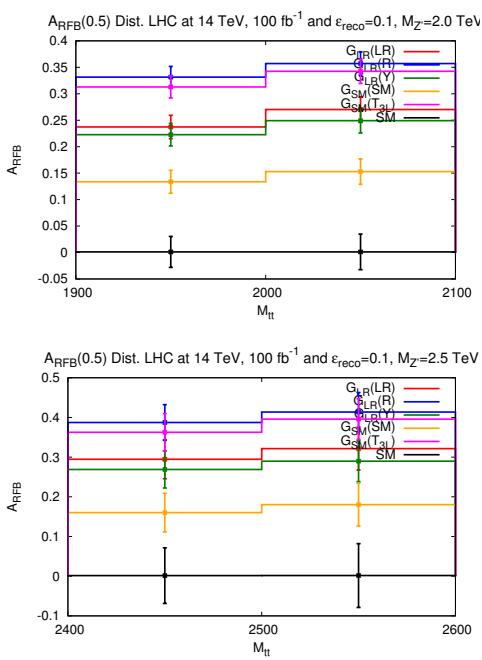

Figure 1: Upper[lower] plots show $A_{L L}$ for the $E_{6}$-type models and $A_{L}$ and $A_{R F B}\left(\left|y_{t t}\right|>0.5\right)$ for the generalised models binned in $M_{t \bar{t}} 100 \mathrm{GeV}$ either side of $M_{Z^{\prime}}=2[2.5] \mathrm{TeV}$ for the LHC at $14 \mathrm{TeV}$ assuming 100 $\mathrm{fb}^{-1}$ of integrated luminosity.

The plots shown in figure 1 of $A_{L L}, A_{L}$ and $A_{R F B}$ distributions are binned in $100 \mathrm{GeV}$ either side of the $Z^{\prime}$ peak. They include statistical uncertainties and fold in an estimated $10 \%$ reconstruction efficiency of the $t \bar{t}$ pair assuming the use of all possible decay channels. Systematic uncertainties may also be important but would require a study beyond parton level. The figures show that the majority of benchmark models can be distinguished from one another using these variables, noting in particular the sensitivity of $A_{L}$ to the relative sign of the vector and axial couplings which allows for a clear distinction between the $G_{S M}$ (sequential) and $G_{L R}$ (left-right symmetric) models in the 'generalised' category as described in equation (2.6).

$A_{L L}$ depends on the couplings in the same way as the total cross section and therefore models that cannot be distinguished in the invariant mass spectrum will remain so in this observable. It is clear that $A_{L}$ is the most powerful observable in that it provides the best distinguishing power along with the extra feature of being sensitive to the handedness of the top couplings. $A_{R F B}$ provides some distinction but the handedness sensitivity is not present for reasons discussed in the previous section. Increasing the $Z^{\prime}$ mass increases the statistical uncertainties but also slightly raises the central values, both as a consequence of the lower SM background. Table 1 is an example of the statistical studies made where the significance of an observable (in this case $A_{L}$ ) is examined assuming 100 $\mathrm{fb}^{-1}$ of integrated luminosity. Here, the significance between various models is a measure of how well they can be distinguished. In almost all cases the significances are important, showing that this variable would certainly be effective in disentangling the 'generalised' models with the discrimi- 
nation decreasing slightly for higher masses. Although not shown in these proceedings, a similar statistical analysis was performed in determining how much integrated luminosity would be required to achieve a significance of $3 \sigma$ between models in the various observables. We showed that in most cases, the models can be distinguished at the relatively early stages of the LHC $\left(\sim 100 \mathrm{fb}^{-1}\right.$ at $14 \mathrm{TeV}$ ) even for the higher mass of $2.5 \mathrm{TeV}$. The cases where this is not possible reflect mostly instances where the couplings are too similar and would be difficult to disentangle.

\begin{tabular}{|c|c|c|c|c|c|c|}
\hline$A_{L}$ & $S M$ & $G L R(L R)$ & $G L R(R)$ & $G L R(Y)$ & $G S M(S M)$ & $G S M\left(T_{3 L}\right)$ \\
\hline$S M$ & - & $31.9(11.1)$ & $40.6(18.3)$ & $30.1(11.2)$ & $22.1(9.8)$ & $38.7(22.5)$ \\
$G L R(L R)$ & $16.9(7.7)$ & - & $10.0(6.6)$ & $2.0(0.1)$ & $62.2(21.7)$ & $81.3(34.5)$ \\
$G L R(R)$ & $21.3(11.5)$ & $4.6(4.0)$ & - & $12.0(6.5)$ & $72.2(30.4)$ & $91.3(44.1)$ \\
$G L R(Y)$ & $16.3(7.8)$ & $1.0(0.1)$ & $5.8(3.9)$ & - & $60.2(21.8)$ & $79.3(34.6)$ \\
$G S M(S M)$ & $11.8(6.3)$ & $33.1(14.8)$ & $38.8(18.8)$ & $33.0(14.9)$ & - & $19.1(13.7)$ \\
$G S M\left(T_{3 L}\right)$ & $20.1(13.9)$ & $42.5(23.0)$ & $48.5(27.2)$ & $42.7(23.2)$ & $9.7(7.8)$ & - \\
\hline
\end{tabular}

Table 1: Significance for $A_{L}$ values around the $Z^{\prime}$ peak of generalised models, for the LHC at $14 \mathrm{TeV}$ only. Upper triangle for $M_{Z^{\prime}}=2.0 \mathrm{TeV}$ and lower triangle for $M_{Z^{\prime}}=2.5 \mathrm{TeV}$. Figures refer to $\Delta M_{t \bar{t}}<100(500)$ $\mathrm{GeV}$.

\section{Conclusion}

We have presented an overview of a phenomenological study on classes of $Z^{\prime}$ models in both spin and spatial asymmetries of $t \bar{t}$ production and showed that there is much scope to observe deviations from the SM and even distinguish between various models, particularly for spin asymmetries. This suggests that the $t \bar{t}$ channel would certainly be a useful complement to the more popular DY channel in the aim of profiling a $Z^{\prime}$ resonance should one be observed in the near future.

It is worth noting that the classes of models studied are benchmarks put forward to set bounds on $Z^{\prime}$ masses best probed in the di-lepton channels. Other models could be better suited to the $t \bar{t}$ channel, such as leptophobic/top-phillic $Z$ 's occurring in composite/multi-site and extra-dimensional models. The profiling techniques discussed in this study would be increasingly more applicable in these scenarios.

Finally, although not addressed in the paper to which these proceedings refer, one can ask whether more can be done in profiling an observed resonance with the view of extracting its fermionic couplings. A previous study attempting to do this in the light lepton sector [12] finds a degeneracy in determining the quark and lepton couplings which can be solved by considering asymmetries in an alternate final state. In a more recent paper [13], we show that asymmetry observables in the $t \bar{t}$ provide independent information which would break this degeneracy and allow for a fit to all couplings in the 'minimal' framework of 5 independent couplings used in many benchmarks.

\section{Acknowledgments}

The work of KM and SM is partially supported through the NExT Institute. LB is supported by the Deutsche Forschungsgemeinschaft through the Research Training Group grant GRK 1102 
Physics of Hadron Accelerators. We would like to thank E. Alvarez for pointing out the discussion on systematic uncertainties. KM would additionally like to thank the organisers of ICHEP 2012 for their financial support.

\section{References}

[1] T. Aaltonen et al. [CDF Collaboration], A Search for high-mass resonances decaying to dimuons at CDF, Phys. Rev. Lett. 102 (2009) 091805 [arXiv:0811.0053 [hep-ex]] and Search for High-Mass $e^{+}$ e-Resonances in p $\bar{p}$ Collisions at $\sqrt{s}=1.96-T e V$, Phys. Rev. Lett. 102 (2009) 031801 [arXiv:0810.2059 [hep-ex]]; V. M. Abazov et al. [Dり Collaboration], Search for Heavy Z' Bosons in the Dielectron Channel with $200 \mathrm{pb}^{-1}$ of Data with the D $\emptyset$ Detector, 4375-Conf and Search for Heavy Z' Bosons in the Dimuon Channel with $250 \mathrm{pb}^{-1}$ of Data with the DØ Detector, 4577-Conf.

[2] G. Aad et al. [ATLAS Collaboration], Search for dilepton resonances in pp collisions at $\sqrt{s}=7 \mathrm{TeV}$ with the ATLAS detector, Phys. Rev. Lett. 107 (2011) 272002 [arXiv:1108.1582 [hep-ex]] and Search for high-mass dilepton resonances with $5 \mathrm{fb}^{-1}$ of pp collisions at $\sqrt{\mathrm{s}}=7 \mathrm{TeV}$ with the ATLAS experiment, ATLAS-CONF-2012-007; S. Chatrchyan et al. [CMS Collaboration], Search for Resonances in the Dilepton Mass Distribution in pp Collisions at $\sqrt{s}=7$ TeV, JHEP 1105 (2011) 093 [arXiv:1103.0981 [hep-ex]] and Search for narrow resonances in dilepton mass spectra in pp collisions at $\sqrt{s}=7 \mathrm{TeV}$, Phys. Lett. B 714 (2012) 158 [arXiv:1206.1849 [hep-ex]].

[3] L. Basso, K. Mimasu and S. Moretti, Z' signals in polarised top-antitop final states, JHEP 1209 (2012) 024 [arXiv:1203.2542 [hep-ph]].

[4] E. Accomando, A. Belyaev, L. Fedeli, S. F. King and C. Shepherd-Themistocleous, Z' physics with early LHC data, Phys. Rev. D 83 (2011) 075012 [arXiv:1010.6058 [hep-ph]].

[5] R. W. Brown, D. Sahdev and K. O. Mikaelian, Probing Higher Order Qcd: Charge Conjugation Asymmetries From Two Gluon Exchange, Phys. Rev. Lett. 43 (1979) 1069; J. H. Kuhn and G. Rodrigo, Charge asymmetry of heavy quarks at hadron colliders, Phys. Rev. D 59 (1999) 054017 [hep-ph/9807420].

[6] M. Arai, N. Okada, K. Smolek and V. Simak, Influence of $Z^{\prime}$ boson on top quark spin correlations at the LHC, Acta Phys. Polon. B 40 (2009) 93 [arXiv:0804.3740 [hep-ph]].

[7] K. Hagiwara and D. Zeppenfeld, Helicity Amplitudes for Heavy Lepton Production in e+ eAnnihilation, Nucl. Phys. B 274 (1986) 1.

[8] H. Murayama, I. Watanabe and K. Hagiwara, HELAS: HELicity amplitude subroutines for Feynman diagram evaluations, KEK-91-11.

[9] T. Stelzer and W. F. Long, Automatic generation of tree level helicity amplitudes, Comput. Phys. Commun. 81 (1994) 357 [hep-ph/9401258].

[10] J. Pumplin, D. R. Stump, J. Huston, H. L. Lai, P. M. Nadolsky and W. K. Tung, New generation of parton distributions with uncertainties from global QCD analysis, JHEP 0207 (2002) 012 [hep-ph/0201195].

[11] G.P. Lepage,Vegas: An Adaptive Multidimensional Integration Program, J. Comp. Phys. 27, 192 (1978) [Erratum, preprint CLNS-80/447, March 1980].

[12] F. Petriello and S. Quackenbush, Measuring Z' couplings at the CERN LHC, Phys. Rev. D 77 (2008) 115004 [arXiv:0801.4389 [hep-ph]].

[13] L. Basso, K. Mimasu and S. Moretti, 'Non-exotic $Z^{\prime}$ signals in $\ell^{+} \ell^{-}, b \bar{b}$ and $t \bar{t}$ final states at the LHC, arXiv:1208.0019 [hep-ph]. 